\title{
Short-Term Learning Induces White Matter Plasticity in the Fornix
}

\author{
Shir Hofstetter, ${ }^{1,2}$ Ido Tavor, ${ }^{2}$ Shimrit Tzur Moryosef, ${ }^{1,2}$ and Yaniv Assaf ${ }^{1,2}$ \\ ${ }^{1}$ Sagol School of Neuroscience and ${ }^{2}$ Department of Neurobiology, George S. Wise Faculty of Life Sciences, Tel Aviv University, 69978 Tel Aviv, Israel
}

\begin{abstract}
Magnetic resonance imaging (MRI) has greatly extended the exploration of neuroplasticity in behaving animals and humans. Imaging studies recently uncovered structural changes that occur in gray and white matter, mainly after long-term training. A recent diffusion tensor imaging (DTI) study showed that training in a car racing game for $2 \mathrm{~h}$ induces changes in the hippocampus and parahippocampal gyri. However, the effect of short-term training on the white matter microstructure is unknown. Here we investigated the influence of short learning tasks on structural plasticity in the white matter, and specifically in the fornix, in humans and rats. Human subjects performed a $2 \mathrm{~h}$ spatial learning task, and rats underwent training for $1 \mathrm{~d}$ in a Morris water maze. Between tasks, subjects were scanned with DTI, a diffusion MRI framework sensitive to tissue microstructure. Using tract-based spatial statistics, we found changes in diffusivity indices in both humans and rats. In both species, changes in diffusion in the fornix were correlated with diffusion changes in the hippocampus, as well as with behavioral measures of improvement in the learning tasks. These results, which provide the first indication of short-term white matter plasticity in the human brain, suggest that the adult brain white matter preserves dynamic characteristics and can be modified by short-term learning experiences. The extent of change in white matter was correlated with their extent in gray matter, suggesting that all components of the neural network are capable of rapid remodeling in response to cognitive experiences.
\end{abstract}

\section{Introduction}

The nervous system possesses an intrinsic ability to learn and adapt to new experiences throughout life (Pascual-Leone et al., 2005). This neural plasticity is manifested both functionally and structurally. Processes underlying cellular mechanisms of structural plasticity include synapse formation (Knott et al., 2002), expansion and retraction of dendritic spines (Hofer et al., 2009; Xu et al., 2009), axonal remodeling (De Paola et al., 2006), astrocyte modifications (Theodosis et al., 2008), and neurogenesis (Ming and Song, 2005). Magnetic resonance imaging (MRI), by allowing in vivo exploration of whole-brain structural changes, provides invaluable information on neuroplasticity. Morphological changes observed in the adult human brains after prolonged training indicate modifications in gray matter volume and cortical thickness (Maguire et al., 2000; Draganski et al., 2004, 2006). Diffusion tensor imaging (DTI), a diffusion MRI framework, is sensitive to the tissue microstructure and yields quantitative indices reflecting micron-scale tissue density and organization (Assaf and Pasternak, 2008).

Morphometric MRI analyses were complemented by DTI studies of nervous system plasticity showing microstructural dif-

Received Sept. 23, 2012; revised May 22, 2013; accepted June 14, 2013.

Author contributions: Y.A. designed research; S.H., I.T., and S.T.M. performed research; S.H. analyzed data; S.H. wrote the paper.

This work was supported by Israel Science Foundation Grant 994/08 and the Future and Emerging Technologies Programme within the Seventh Framework Programme for Research of the European Commission (FET-Open "CONNECT" Project Grant 238292).

The authors declare no competing financial interests.

Correspondence should be addressed to Prof. Yaniv Assaf, Department of Neurobiology, George S. Wise Faculty of Life Science, Tel Aviv University, 69978 Tel Aviv, Israel. E-mail: assafyan@post.tau.ac.il.

DOI:10.1523/JNEUROSCI.4520-12.2013

Copyright $\odot 2013$ the authors $\quad 0270-6474 / 13 / 3312844-07 \$ 15.00 / 0$ ferences between nerve fiber tracts of experts and controls (Bengtsson et al., 2005; Imfeld et al., 2009) or before and after long-term training (weeks to months) (Scholz et al., 2009; Takeuchi et al., 2010). However, studies of the short-term training effect on tissue microstructure are scarce. In one recent study, changes in diffusion indices in gray matter were detected after only $2 \mathrm{~h}$ of training, pointing to a rapid timescale of structural plasticity (Sagi et al., 2012). However, it is still unknown whether short learning experiences can also modify adult brain white matter. Recent in vitro studies provided evidence for an effect, after only a few hours, of electrical activity on myelination (Wake et al., 2011), as well as for oligodendrocyte development and differentiation into myelinating oligodendrocytes (Stevens et al., 2002).

Here we used DTI to examine structural changes in white matter tracts after performance of short spatial-learning tasks by humans and rats. Human subjects were trained for $2 \mathrm{~h}$ in a car racing game, a task found previously to induce changes in diffusion in the hippocampus and parahippocampal gyrus (Sagi et al., 2012). The rat study was based on a short version of Morris water-maze training. Performance in a maze engages the fimbria-fornix (Sutherland and Rodriguez, 1989; de Bruin et al., 2001), and training induces changes in the hippocampal formation (Blumenfeld-Katzir et al., 2011; Lerch et al., 2011). Our analysis was restricted to the fornix, which is the main tract connecting the hippocampus to the medial diencephalon, an area suggested to be involved in memory processes (Gaffan and Gaffan, 1991; Rudebeck et al., 2009). Because the alignment of the whole-brain images used in the above studies was not sufficient to analyze white matter across subjects, we applied tract-based spatial statistics (TBSS), a method used commonly to study white matter (Smith et al., 2006). 

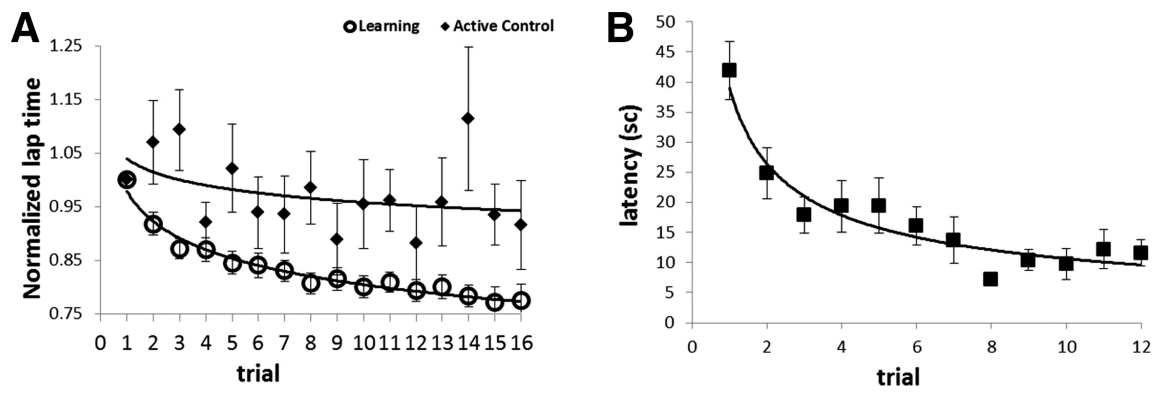

Figure 1. $\boldsymbol{A}$, Improvement in the car racing game. Normalized lap times (mean $\pm \mathrm{SEM}$ ) show significant reduction in time required to complete the track in the LG group $(n=33)$ but not in the AC group $(n=13)$. $\boldsymbol{B}$, Improvement in the water-maze task shown by the reduction in the time required to reach the hidden platform (mean \pm SEM) $(n=19)$. This figure is based on data presented by Sagi et al. (2012), excluding subjects that were not part of the analyses in the current study.

\section{Materials and Methods}

\section{Human study}

Subjects. The study participants were 70 adult right-handed volunteers (39 women and 41 men; mean \pm SD age, $26.6 \pm 3.8$ years; range, $20-38$ years). The subjects were divided into three experimental groups: (1) a learning group (LG; $n=40,17$ males and 23 females; mean age, 26.7 years); (2) a nonspatial learning group that served as an active control (AC; $n=15,7$ males and 8 females; mean age, 26.9 years); and (3) a passive control group (PC; $n=15,7$ males and 8 females; mean age, 26.1 years). This dataset includes subjects from the study by Sagi et al. (2012), as well as a new cohort of subjects. The research protocol was approved by the Institutional Review Board of the Tel Aviv Sourasky Medical Center. Each participant signed a written informed consent form. None of them had a history of neurological disease, psychological disorders, drug or alcohol abuse, or use of neuropsychiatric medication. All had intact vision.

Behavioral paradigm. Subjects in the LG and AC groups were trained to play a computer car racing game (The Need for Speed; Electronic Arts). The LG training consisted of completing 16 laps (trials) divided into four training sessions (four laps each). The same track was presented in each trial. The aim was to learn the track and achieve better lap times. At the end of each training session, subjects were asked to sketch an outline of the track and were given snapshots of locations along the track that they had to arrange in the correct order. In the AC group, subjects played the same car racing game for the same duration as the LG subjects, but in each lap (trial) the track was different. Subjects in the PC group, like those in the other two groups, underwent two MRI scan, $2 \mathrm{~h}$ apart, but unlike the others, they did not participate in any training tasks between the imaging sessions.

Imaging data. Data acquisition was performed at the Tel Aviv Sourasky Medical Center with a 3 T MRI system (GE Healthcare). Each subject underwent two imaging sessions, $\sim 2 \mathrm{~h}$ apart. For both the LG and AC subjects, these scanning sessions took place before and immediately after performance of their assigned task. The MRI protocol of the first scanning session included both conventional anatomy sequences and DTI acquired with an eight-channel head coil. In the second scanning session, only DTI scans were administered.

The DTI protocol included acquisition of whole-brain diffusionweighted images with the following parameters: $2.1 \times 2.1 \times 2.1 \mathrm{~mm}^{3}$ resolution reconstructed to $1.58 \times 1.58 \times 2.1 \mathrm{~mm}^{3}$, up to 70 axial slices, $\Delta / \delta=33 / 26 \mathrm{~ms}, b=1000 \mathrm{~s} / \mathrm{mm}^{2}$, with 19 gradient directions and an additional image with no diffusion weighting $\left(b_{0}\right.$ image). To increase the signal/noise ratio, the DTI scan was repeated three times.

To correct for head motion, we used a least-squares algorithm and six-parameter (rigid-body) transformations using SPM (version 2; University College London). DTI was analyzed by the DiVa (diffusion imaging visualization and analysis) software (Pasternak et al., 2008, 2009) implemented in MATLAB 7.3.0 (MathWorks), from which maps of fractional anisotropy (FA), mean diffusivity (MD), principal (axial) diffusivity $(\lambda 1)$, and radial diffusivity (RD) were computed.

Analysis of DTI data. The data were subjected to voxelwise statistical analysis by TBSS (Smith et al., 2006), part of the FSL [for FMRIB Software Library (in which FMRIB is Functional MRI of the Brain, Oxford
University, Oxford, UK)] package (Smith et al., 2004). Briefly, FA images from both scans for each subject were first nonlinearly aligned into to a $1 \times 1 \times 1 \mathrm{~mm}$ common space using the nonlinear image registration tool of FMRIB. Two LG subjects were excluded because their ventricles were enlarged, causing misalignment of the fornix tract after nonlinear normalization. The transformed maps were averaged to generate a mean FA image, which was then "thinned" to create a white matter FA skeleton at a threshold FA value of 0.2 . The aligned FA image of each subject was then projected onto the mean FA skeleton. The MD, $\lambda 1$, and RD maps were analyzed by application of the same spatial transformation parameters as those estimated in the initial FA analysis. The course of the fornix in the ventricles makes it prone to contamination by CSF. To minimize artifacts resulting from partial volume effects, we excluded the data from subjects in whom $>10 \%$ of the voxels in the fornix skeleton had MD values, indicating CSF contamination $(\mathrm{MD}>2)$. Accordingly, five subjects were excluded from the $\mathrm{LG}$, two from the AC, and six from the PC groups.

In view of our hypothesis that the fornix plays a role in this short-term learning, we used anatomical atlases to create a mean fornix mask for statistical analysis. Our focus on analyzing the fornix tract instead of the whole-brain skeleton also helped to reduce multiple comparisons issues and to limit false-positive errors.

\section{Rat study}

Behavioral paradigm. For this part of the study, 44 male Wistar rats (4 months old) underwent two MRI scans during the light phase, $1 \mathrm{~d}$ apart. All rats were maintained on a $12 \mathrm{~h}$ light/dark cycle with access to food and water ad libitum. Before scanning, the rats were anesthetized with 1-2\% isoflurane in oxygen.

The rats were divided into three groups: (1) a learning group ( $\mathrm{L} ; n=$ $24)$; (2) a cued (visible platform) swim group ( ; $n=10$ ); and (3) a passive (naive) control group $(\mathrm{N} ; n=10)$. This dataset was included in the study by Sagi et al. (2012). Between scans, the rats in group L were trained to perform a short water-maze task in a 120 -cm-diameter pool. Training consisted of three repeats of four trials, with $\sim 40$ min between trials. At each trial, the rat was placed in a different quadrant of the pool and had to find, based on spatial cues, the location of a hidden platform. An HVS video-tracking system (HVS Image) was used to record the time taken to reach the platform, the time spent in each quadrant, and the swimming speed. Rats in group $\mathrm{C}$ were given the same task, but the platform was made visible by an object placed on top of it. The rats in group $\mathrm{N}$ remained in their cages between the two scans.

Imaging data. MRI scanning was performed using a 7 T MRI scanner (Bruker) with a $30 \mathrm{~cm}$ bore and a gradient strength of up to $400 \mathrm{mT} / \mathrm{m}$. The MRI protocol included (DTI) acquisition with a diffusion-weighted spin-echo echo-planar-imaging pulse sequence. Acquired volumes were 21 slices, each $0.75 \mathrm{~mm}$ thick with the following parameters: resolution of $0.2 \times 0.2 \mathrm{~mm}^{2}$ (matrix size, $128 \times 128$; field of view, $25.6 \mathrm{~mm}^{2}$ ), repetition time of $4000 \mathrm{~ms}$, echo time of $25 \mathrm{~ms}, \Delta / \delta=10 / 4.5 \mathrm{~ms}$, four echo-planar-imaging segments, and 15 noncollinear gradient directions with a single $b$-value shell at $1000 \mathrm{~s} / \mathrm{mm}^{2}$ and one image with a $b$ value of $0 \mathrm{~s} / \mathrm{mm}^{2}\left(b_{0}\right.$ image). The imaging protocol was repeated three times for signal averaging and to compensate for acquisition in which significant head motion was observed. Each DTI acquisition took $4.5 \mathrm{~min}$, and the entire MRI protocol lasted $\sim 20 \mathrm{~min}$.

Because excessive breathing during DTI acquisition can lead to motion artifacts in the sampled slice, images of every slice at each gradient direction were screened automatically for motion artifacts. If the signal in the outer borders of the brain was significant, the slice was omitted from analysis. DTI acquisition was repeated three times to compensate for the extracted slices. DTI analysis was performed with in-house software, implemented on MATLAB, and FA and MD maps were produced. 

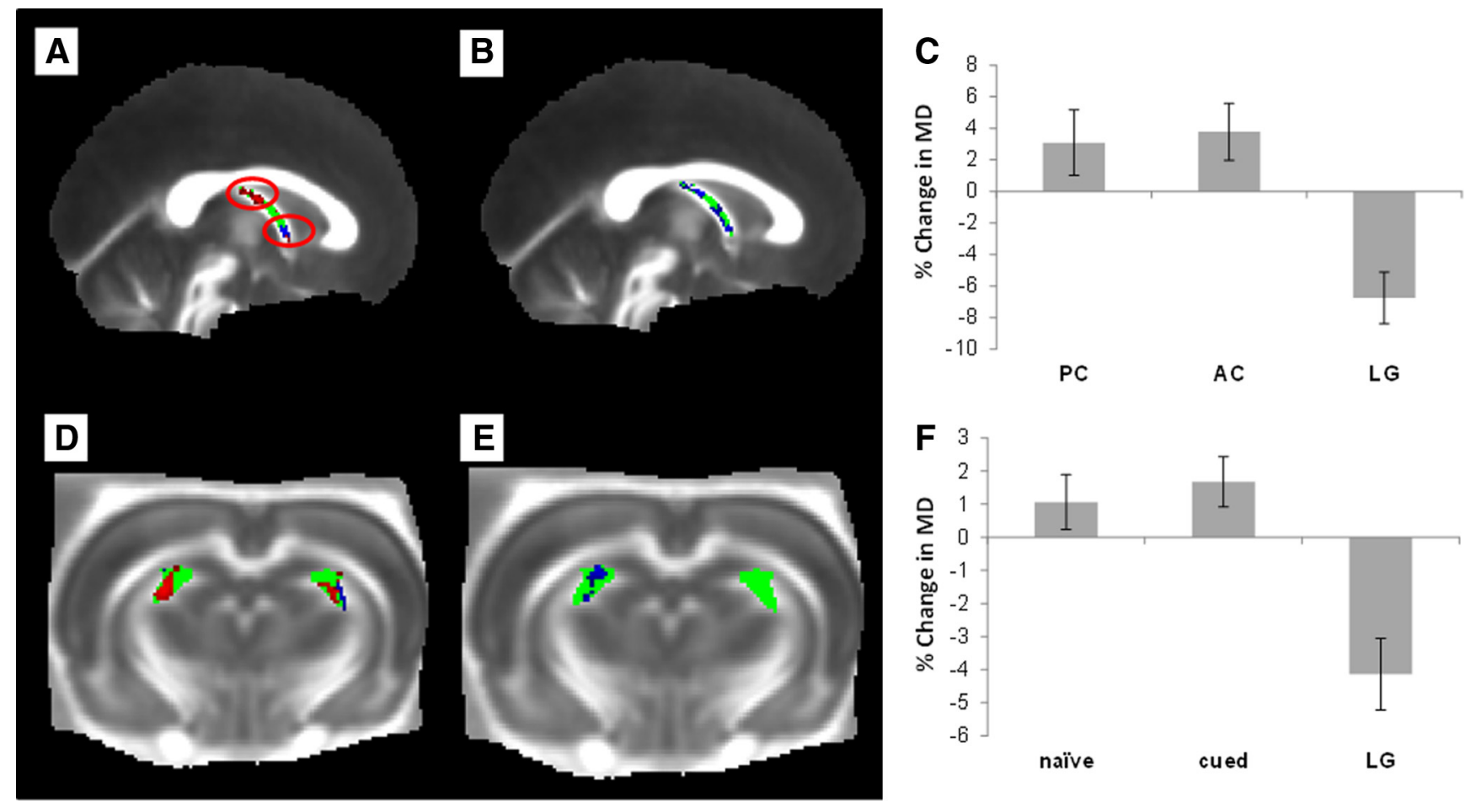

Figure 2. Changes in diffusion indices after short-term learning tasks. Statistical images were threshold at $p<0.05$ for display purposes. $A$, Reduction in MD in the two learning subgroups as identified by TBSS. Statistical images are overlaid on the mean FA image of all subjects and the fornix skeleton mask (green). Red clusters show reduction in MD in LG group. Blue clusters display reduction in MD in the second subgroup, LG2. $\boldsymbol{B}$, Reduction in $\lambda 1$ (blues voxels) is presented, overlaid on the fornix skeleton mask (green) and the men FA image. $\boldsymbol{C}$, Mean values of the percentage of change in MD revealed by the group $\times$ time interaction analysis between the learning group and the two control groups $(p<0.01$ clusters $\geq 5)$. $D$, Reduction in MD in the two rat subgroups after $1 \mathrm{~d}$ training in the Morris water maze. Red clusters present regions in which reduction in MD in the L group was found, and blue clusters display the reduction in L2. Results are superimposed on the fornix skeleton (green) and the mean FA rat image. $E$, Reduction in L1 in the L group, overlaid on the fornix skeleton (green) and the mean FA map. F, Mean values of the percentage of change in MD in the analysis across groups $(p<0.01)$.

Image normalization was performed using SPM2. Each rat was normalized to a rat atlas template that was created from one representative rat and included $b_{0}$ and FA images registered with a digitized version of the Paxinos and Watson (2005) stereotaxic atlas. The normalization procedure initially included bias correction of all $b_{0}$ images and coregistration of each $b_{0}$ image with the $b_{0}$ template using a six-parameter rigidbody transformation. The parameters of the coregistration were applied on the different FA and MD maps. The coregistered FA maps were then normalized to the FA template using a 12-parameter affine nonlinear transformation and $0.2 \mathrm{~mm}$ smoothing. Normalization parameters were applied on all DTI-indexed maps (FA, MD, $\lambda 1$, and RD). After the normalization, data were reexamined, and some rats were excluded because of motion artifacts, asymmetrical ventricles, or Nyquist ghost artifacts in relevant brain areas. Five rats were omitted from group L, three from group C, and four from group $\mathrm{N}$.

A mean FA map was created from the normalized images, and a skeleton was generated from it using the TBSS procedure, with a threshold at $F A \geq 0.1$. Rat FA data were projected onto the skeleton. Projection of $\mathrm{MD}, \lambda 1$, and RD values to the skeleton was also applied. Statistical analysis was restricted to the fornix by use of a mean fornix mask that was created using the Paxinos and Watson (2005) stereotaxic atlas.

Statistical analyses. To ascertain fair comparison across groups and to allow the replication of results, the learning groups in both the human and rat studies were divided into two subgroups. Division of the learning groups in this way helped to reduce the difference in sample size between the learning groups and the control and provided an additional cohort of subjects that could be independently analyzed while also serving as a replication group for the learning effect. Such replication strengthens the inference derived from the results of the statistical analysis.

The learning group in the human study was divided on the basis of the analysis described by Sagi et al. (2012). The first learning cohort (LG1) was compared with both control groups (LG1, $n=14$; AC, $n=13$; PC, $n=9$ ). The second learning cohort (termed LG2 in this study; $n=19$ ) comprised the group used by Sagi et al. (2012) for replication with additional new subjects who underwent the same experimental procedure.

The learning group in the rats study was randomly divided into two. One group (L1) was compared with the $\mathrm{C}$ and $\mathrm{N}$ groups (L1, $n=10 ; \mathrm{C}$, $n=7 ; \mathrm{N}, n=6)$, and the other was used for replication (L2, $n=9)$. We report on changes in diffusion indices that pass $p<0.01$ in the across groups analyses (learning vs controls), as well as in the replication group.

Data both in the human and rat study were statistically analyzed using the Randomize program (part of FSL), carrying voxel-by-voxel nonparametric permutation test (5000 permutations) and thresholded using threshold-free cluster enhancement, which obviates the use of an arbitrary threshold for the initial cluster formation (Smith and Nichols, 2009). General linear model designs were applied for analysis of group contrasts $\times$ time interaction, by paired $t$ test analysis for the replication groups, and by linear correlations with changes in gray matter (hippocampus) and behavioral measurements.

To examine whether changes in the fornix were correlated with changes in the hippocampus, we conducted paired $t$ tests on the normalized MD maps of the learning groups of both humans and rats, using SPM2 (for the normalization process on the human images, see Sagi et al., 2012). Mean values of MD changes in the cluster of results found in the hippocampus were extracted for each subject, and the percentage of change in MD was calculated. These values were applied in a voxel-based correlation analysis with the differences (post-task vs pre-task) in diffusion indices in the fornix.

\section{Results}

Behavioral results

In the human study, improvement in the learning task was demonstrated by all LG subjects, as indicated by a reduction of $22.5 \pm$ 

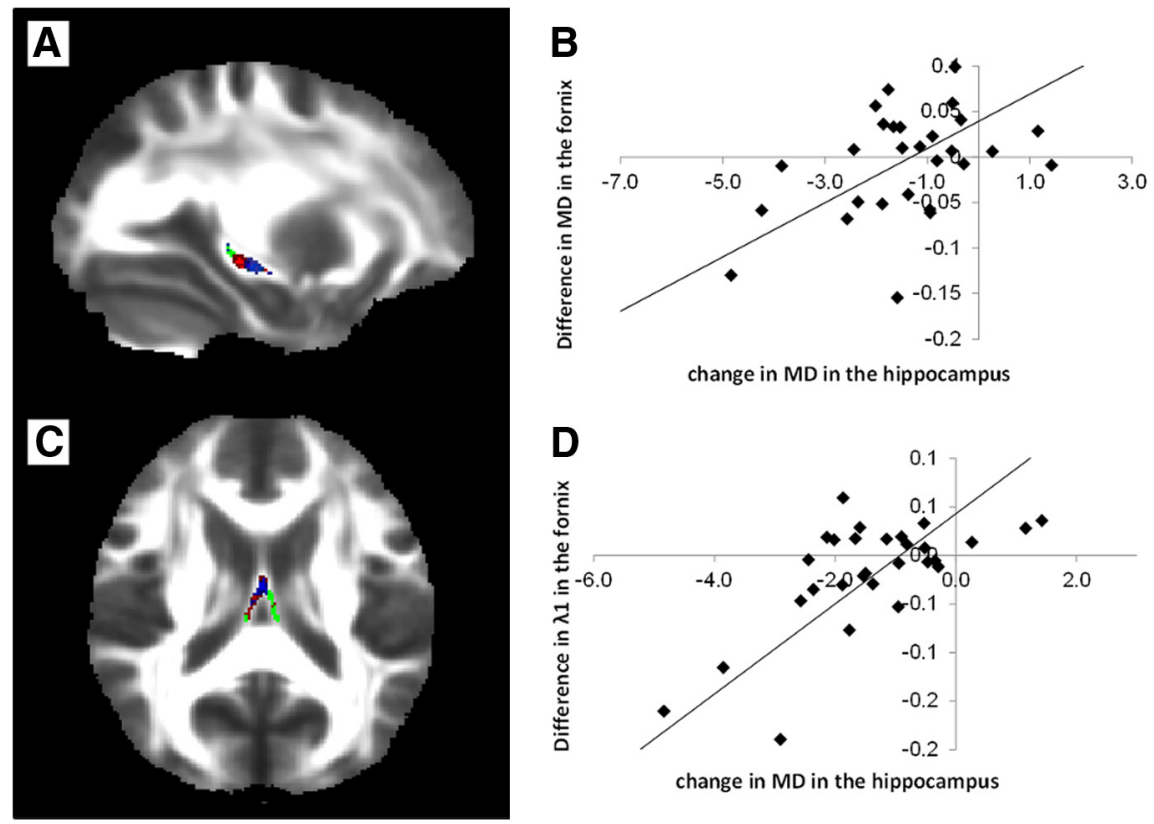

Figure 3. Correlation between changes in gray matter (hippocampus) and the difference in diffusion in the fornix. $\boldsymbol{A}$, Statistical maps showing correlation of MD (red) and $\lambda 1$ (blue) with the percentage of change in the hippocampus are thresholded at $p<0.005$ and overlaid on the fornix mask (green) and the mean FA map. $\boldsymbol{B}$, Correlation analysis of the difference in MD values with the percentage of change in the hippocampus $(p<0.05$, corrected; $r=0.62)$. $C$, Colocalization of regions in which decrease in MD was found (red; $p<0.05$ for display purposes) and correlation analysis of the difference in $\lambda 1$ with changes in MD the hippocampus (blue; $p<0.005$ ), overlaid on the fornix skeleton mask (green) and mean FA image. $\boldsymbol{D}$, Correlation analysis of the difference in $\lambda 1$ values with the percentage of change in MD in the hippocampus ( $p<0.05$, corrected; $r=0.87)$.
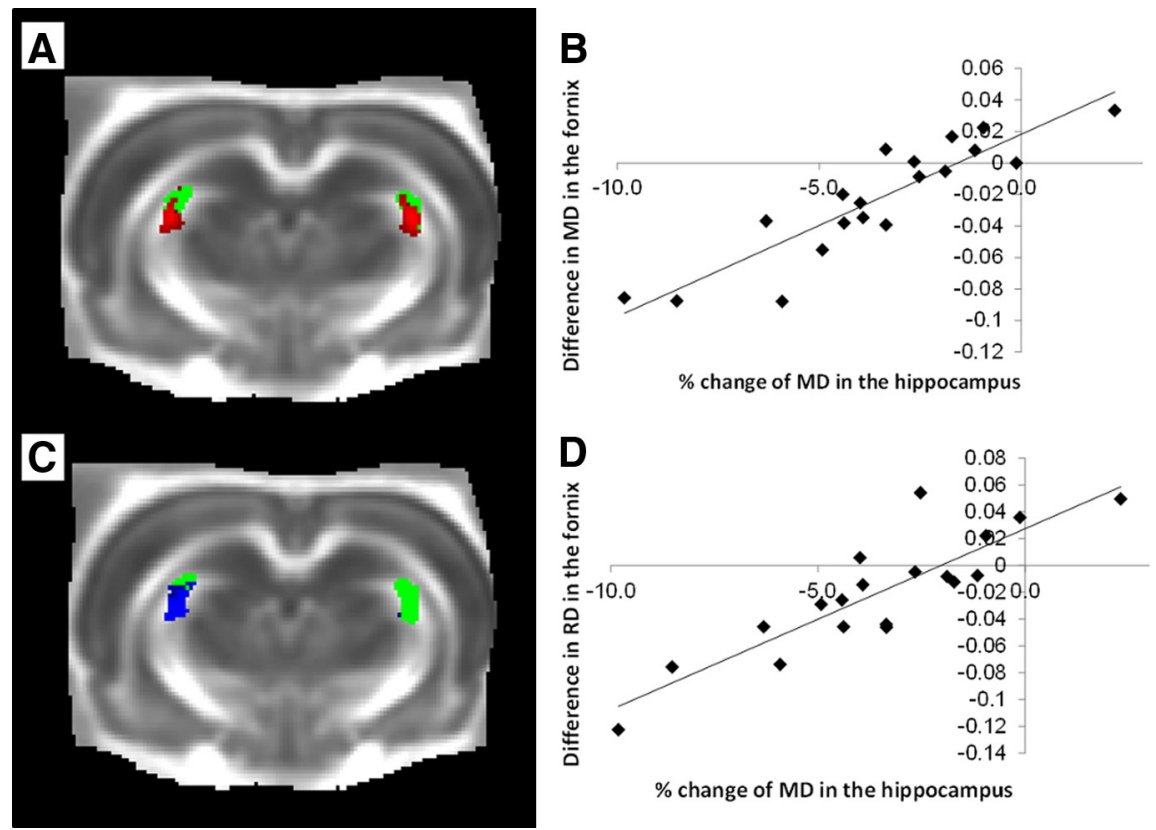

Figure 4. Correlation analysis between changes in MD in the hippocampus of rats undergoing $1 \mathrm{~d}$ of water-maze training with the change in diffusion indices in the fornix. $A$, Regions in which positive correlation between changes in MD in the fornix and the percentage of change in MD in the hippocampus (red; $p<0.005$ ) are superimposed on the fornix skeleton mask (green) and mean FA image. $\boldsymbol{B}$, Correlation analysis of the difference in $M D$ in the fornix and the percentage of change in MD in the hippocampus ( $p<0.05$, corrected; $r=0.9)$. C, A positive correlation was also found between the difference in $\mathrm{RD}$ and the percentage of change in $\mathrm{MD}$ in the hippocampus (blue; $p<0.005$ ), overlaid on the fornix skeleton mask (green) and mean FA image. $\boldsymbol{D}$, Correlation analysis between the difference in $\mathrm{RD}$ and the percentage of change in MD in the hippocampus ( $p<0.05$, corrected; $r=0.87$ ).
$3 \%($ mean \pm SEM, $p<0.0001 ; n=33)$ in their normalized lap times and an improvement in their scores for picture arrangement $(p<0.0001$; Fig. $1 A)$. In contrast, the AC group showed no improvement in their normalized lap time (Fig. 1A). In the rat study, latency time of the $\mathrm{L}$ group rats in the water maze were significantly reduced $(57.4 \pm 9.4 \%$, mean \pm SEM, $p<0.0001 ; n=19$; Fig. 1B).

\section{Short-term structural changes in the fornix}

To assess the effect of learning on structural changes in the fornix, we examined group (learning vs two control groups) $X$ time (scan time) interaction. We did this by looking for clusters in which changes in diffusion indices were significantly different in the LG than in both of the control groups. Significant differences in MD were found in two clusters in the body of the fornix ( $p<0.01$ cluster $\geq 5$, uncorrected; at most significant voxel, $p=$ 0.001). In these clusters, MD in LG1 was decreased by an average \pm SEM of $6.75 \pm$ $1.64 \%$ (Fig. $2 A, C$ ). Reduction in MD was also found in LG2 in the column of the fornix ( $p<0.05$ cluster $\geq 5$, uncorrected; at most significant voxel, $p=0.006$; Fig. $2 A$ ). To confirm that voxels showing significant effect were indeed located within the tract, significant clusters were deprojected (using an FSL de-project tool) back to each subject's standard-space (nonlinearly registered) FA images.

A decrease in FA was found in the analysis across the three groups. However, the same change was not observed at the same statistical threshold in the LG2, in which a decrease was found only at $p<0.05$. However, additional analysis revealed that the observed reduction in $\mathrm{MD}$ derived from reduction in the $\lambda 1(p<0.01$ cluster $\geq 5$; Fig. $2 B$ ) but not from changes in the RD. Reduction in $\lambda 1$ was detected in the same regions as those showing reductions in MD.

In the rat study, analyses of the three groups $(\mathrm{L}$ vs $\mathrm{C}$ and $\mathrm{N}) \times$ time interaction disclosed a similar pattern of change in $\mathrm{MD}$ as was found in the human study. An average \pm SEM decrease of $5 \pm 1.08 \%$ in MD was found in group L1 (Fig. 2D,F). This reduction was also observed in the replication group (L2) $(p<0.01$ cluster $\geq$ 4 , uncorrected; at most significant voxel, $p=0.002$; Fig. $2 D$ ). Additional analysis revealed that the decrease in MD originates from a reduction in $\lambda 1(p<0.01$ cluster $\geq 3$; Fig. $2 E$ ). In both analyses, a decrease in FA was found at less strict $p$ values of $p<0.05$. 
Correlation with structural changes in the gray matter of the hippocampus Previous whole-brain analyses of the learning groups in both human and rat studies revealed significant reductions in $\mathrm{MD}$ in the hippocampus (Sagi et al., 2012). In the present study, we found that this change in gray matter in the hippocampus is correlated with the difference between pre-task and post-task diffusion indices in the fornix. In the human study, changes in MD values in the hippocampus was correlated with the difference in $\mathrm{MD}$ values in the fornix (Fig. $3 A, B ; p<0.05$, corrected; $r=0.62$ ), as well as with the difference in pre-task and post-task $\lambda 1$ values in the fornix (Fig. $3 A, C, D ; p<0.05$, corrected; $r=0.87$ ). Correlation between change in MD in the hippocampus and change in $\lambda 1$ in the fornix was found in the left and right crus of the fornix, as well as in the body of the fornix in which we found a significant reduction in MD. In the rat study, change in $\mathrm{MD}$ in the hippocampus was also correlated with the differences between MD values in the fornix (Fig. $4 A, B ; p<0.05$, corrected; $r=0.9$ ) and with the difference between RD values in the fornix (Fig. $4 C, D ; p<0.05$, corrected; $r=0.87$ ).

\section{Correlation with behavioral data}

We also analyzed the correlation between behavioral measures and differences in diffusion indices. The rate of improvement in the car racing game was found to correlate with reductions in $\mathrm{MD}(p<$ 0.005 cluster $\geq 5 ; r=0.55$ ) and FA (Fig. $5 A, B ; p<0.005$ cluster $\geq 5 ; r=-0.74)$ in the column and right crus of the fornix. Significant correlations were also found between the averaged picture scores and differences in $\mathrm{MD}$ (Fig. $5 C, D ; p<0.005$ cluster $\geq 5 ; r=0.52)$ and $\lambda 1(p<0.005$ cluster $\geq 5 ; r=0.53$ ) in the body of the fornix.

One measure of improvement in the water-maze paradigm is a reduction in the time required for the rat to reach the platform. This learning measure was found to be correlated with changes in MD in the fornix ( $p<0.005$ cluster $\geq 5 ; r=0.58)$, as well as with $\mathrm{RD}$ differences in the fornix (Fig. $5 E, F ; p<0.005$ cluster $\geq 5 ; r=0.78$ ).

\section{Discussion}

Our results provide the first indication of short-term white matter plasticity in the human brain. Using diffusion MRI, we showed that short periods of spatial learning induce changes in diffusion indices in the fornix. We found that these changes exist across species and are related to coextending changes in diffusion in gray matter and correlated with behavioral learning progression and improvement.

The hypothesis put forward in this study was that microstructural changes occur in the white matter after short learning experiences and that the rapidly achieved structural plasticity can be

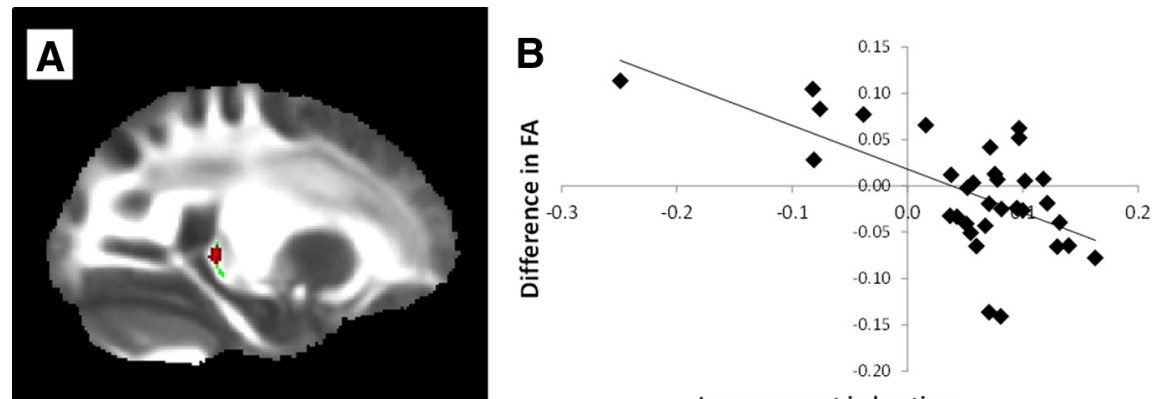

Improvement in lap time
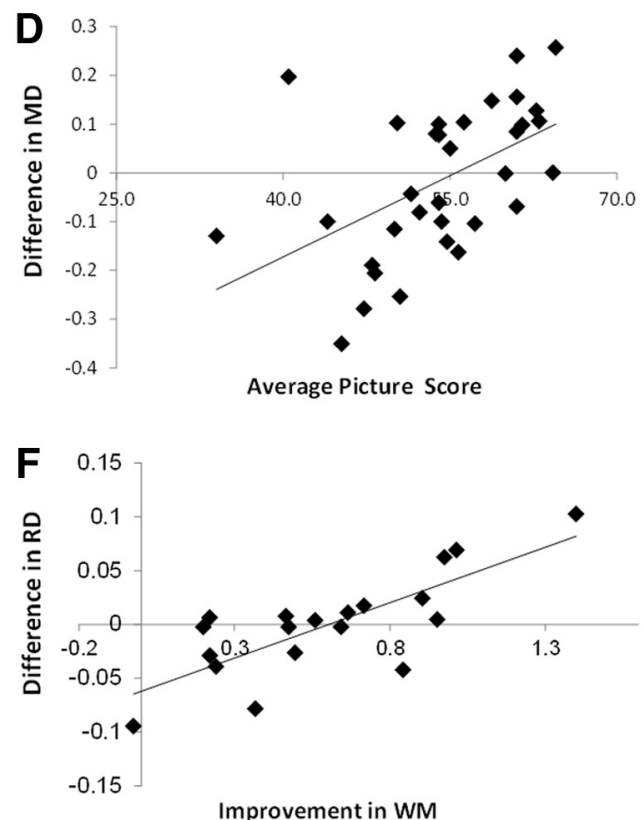

Improvement in WM

Figure 5. Correlation analysis between changes in diffusion indices in the fornix and behavioral measurements. $\boldsymbol{A}$, Regions in which changes in FA were found to correlate with improvement in the car racing game (red; $p<0.005$ ), overlaid on the fornix mask (green) and mean FA image. $\boldsymbol{B}$, Correlation analysis of change in FA in the fornix and improvement in lap time $(p<0.005 ; r=$ , colocalized between changes in MD in the fornix and averaged picture score $(p<0.005 ; r=0.52)$. $\boldsymbol{E}$, Blue clusters present regions in which correlation between changes in RD in the fornix and improvement in the Morris water maze ( $p<0.05$ for display purposes) was found, overlaid on the fornix skeleton mask (green) and mean FA image. $\boldsymbol{F}$, Correlation analysis between changes in RD and improvement in Morris water maze $(p<0.005 ; r=0.78)$.

detected by DTI. This imaging technique yields quantitative measurements of white matter microstructure and can therefore be used to study plasticity in vivo (Scholz et al., 2009; BlumenfeldKatzir et al., 2011; Lerch et al., 2011). Studies have indeed demonstrated anatomical differences in the white matter of groups of experts, such as musicians (Imfeld et al., 2009), as well as correlations between fiber tract organization and the amount of piano practice (Bengtsson et al., 2005), and changes in diffusion after training in juggling (Scholz et al., 2009) or after meditating (Tang et al., 2010). Our study further affirms the utility of diffusion imaging in neuroplasticity research and presents new evidence for the temporal progression of structural plasticity in white matter within short periods of time. In agreement with studies such as those mentioned above, we suggest that white matter in the adult brain preserves dynamic characteristics. It seems clear that biochemical and electrophysiological changes (see below and Wake et al., 2011) can induce rapid structural remodeling in the white matter in the form and content of myelin and other cellular structures. 
The findings on changes in diffusion in the fornix support and complement a previous research on short-term plasticity that revealed a decrease in MD in the human hippocampus and parahippocampal gyrus after the same spatial learning task as the one used in the present work. We showed here that those gray matter modifications correlate with structural changes in the fornix, the main hippocampal projection. A similar strong correlation between changes in MD in the fornix and changes in the hippocampus was found in our rat study. Together, these findings raise the possibility that microstructural alterations occur in related gray and white matter and that the extent of change they undergo is associated. These correlations further point to a connected physiology of the neural network, in which all of its components interact and become modified by new experiences. Although MRI does not reveal the starting point of these changes or indicate whether changes in one component generate changes in others, it does suggest that a comprehensive study of neuroplasticity should incorporate scrutiny of both gray and white matter. Furthermore, changes in diffusion indices in the fornix, in both humans and rats, were found to correlate with improvement in the learning procedures. Therefore, it seems that our aptitude to learn is connected to the biological processes underling learning progression. In addition, the observed correlation between the mean picture scores that subjects achieved in the car racing task and the changes in diffusion in the fornix supports a role for the fornix in memory and recollection processes (Rudebeck et al., 2009).

It should be noted that the results showing a decrease in MD and $\lambda 1$ in the fornix in learning task participants did not pass multiple comparisons. Structural changes in white matter induced over a short periods (hours) would not be expected to produce extreme modifications in the tissue and would be expected, as a consequence, to be buried in noise. However, the same results showing a decrease in MD emerged from the two separate analyses that were conducted in both the human and rat studies. In addition, results of the correlation analyses further support a role for the fornix in learning-induced plasticity. Aside from demonstrating rapid attainment of plasticity of the white matter, this work underscores the advantages of using a tractbased approach in the investigation of white matter. Whole-brain voxel-based analysis without track registration failed to provide adequate alignment of the relevant tracts to enable proper voxelbased comparisons within and between subjects.

Interpretation of the changes in diffusion in white matter is not a straightforward matter. MD and FA are generally believed to depict tissue density and fiber organization, respectively. Reduction in MD might be attributed to changes in extracellular volume (Ransom et al., 1985; Sykova, 1997), swelling of cells (Le Bihan, 2007), or an increase in glia cell volume (Kleim et al., 2007; Theodosis et al., 2008; Markham et al., 2009). Electrical activity might also induce structural changes in axons. Swelling of axons was found in association with action potentials (Iwasa and Tasaki, 1980; Iwasa et al., 1980; Tasaki and Byrne, 1990). Activated axons transmit signals to neighboring glial cells, thereby promoting myelination (Ishibashi et al., 2006; Wake et al., 2011). It could be postulated that this axon-to-glia signaling promote changes in glial cell morphology. Remodeling of astrocytic processes was found to occur after training in the water maze (BlumenfeldKatzir et al., 2011; Lerch et al., 2011; Sagi et al., 2012) . In addition, oligodendrocyte maturation and morphology were found to be altered as a result of electrical activity in axons (Stevens et al., 2002). Changes in cell size and the numerous cellular processes in glia may result in a decrease in $\lambda 1$. Additional research is needed to reveal the biological processes that underlie learning-induced plasticity in white matter and their relationship to diffusion parameters. Although DTI represents a state-of-the-art method for studying white matter, alternative techniques using diffusion MRI were recently suggested. Methods based on models such as CHARMED (for composite hindered and restricted model of diffusion) (Assaf et al., 2004), AxCaliber (Assaf et al., 2008), and ActiveAx (Alexander et al., 2010) have the advantage of quantifying the contribution of different tissue components to the signal (e.g., axonal compartment and axonal composition). These methods allow direct examination of specific tissue components. This may be helpful in determining the biological origins of white matter plasticity and should be applied in future studies.

To summarize, this study revealed changes in diffusion in the fornix of adult humans and rats after only $2 \mathrm{~h}$ of training in a learning task, as well as marked correlations between behavior and structural changes in the fornix origin, the hippocampus. Because diffusion indices are indicative of tissues microstructure, the results suggest that short-term learning can induce structural plasticity in the white matter. Additional research is needed to understand the cellular processes that underlie these changes, as well as the time course of their development. This study suggests that the multiple components of the neural network constitute a dynamic system that is able to achieve rapid remodeling as a result of cognitive experiences. An additional striking finding is the ability of MRI to map and quantify micron-scale processes in the neural tissue, offering the opportunity to study the physiology of the human brain in different timescales and under various spatial conditions.

\section{References}

Alexander DC, Hubbard PL, Hall MG, Moore EA, Ptito M, Parker GJ, Dyrby TB (2010) Orientationally invariant indices of axon diameter and density from diffusion MRI. Neuroimage 52:1374-1389. CrossRef Medline

Assaf Y, Pasternak O (2008) Diffusion tensor imaging (DTI)-based white matter mapping in brain research: a review. J Mol Neurosci 34:51-61. CrossRef Medline

Assaf Y, Freidlin RZ, Rohde GK, Basser PJ (2004) New modeling and experimental framework to characterize hindered and restricted water diffusion in brain white matter. Magn Reson Med 52:965-978. CrossRef Medline

Assaf Y, Blumenfeld-Katzir T, Yovel Y, Basser PJ (2008) AxCaliber: a method for measuring axon diameter distribution from diffusion MRI. Magn Reson Med 59:1347-1354. CrossRef Medline

Bengtsson SL, Nagy Z, Skare S, Forsman L, Forssberg H, Ullén F (2005) Extensive piano practicing has regionally specific effects on white matter development. Nat Neurosci 8:1148-1150. CrossRef Medline

Blumenfeld-Katzir T, Pasternak O, Dagan M, Assaf Y (2011) Diffusion MRI of structural brain plasticity induced by a learning and memory task. PloS One 6:e20678. CrossRef Medline

de Bruin JP, Moita MP, de Brabander HM, Joosten RN (2001) Place and response learning of rats in a Morris water maze: differential effects of fimbria fornix and medial prefrontal cortex lesions. Neurobiol Learn Mem 75:164-178. CrossRef Medline

De Paola V, Holtmaat A, Knott G, Song S, Wilbrecht L, Caroni P, Svoboda K (2006) Cell type-specific structural plasticity of axonal branches and boutons in the adult neocortex. Neuron 49:861-875. CrossRef Medline

Draganski B, Gaser C, Busch V, Schuierer G, Bogdahn U, May A (2004) Neuroplasticity: changes in grey matter induced by training. Nature 427 : 311-312. CrossRef Medline

Draganski B, Gaser C, Kempermann G, Kuhn HG, Winkler J, Büchel C, May A (2006) Temporal and spatial dynamics of brain structure changes during extensive learning. J Neurosci 26:6314-6317. CrossRef Medline

Gaffan D, Gaffan EA (1991) Amnesia in man following transection of the fornix. Brain 114:2611-2618. CrossRef Medline

Hofer SB, Mrsic-Flogel TD, Bonhoeffer T, Hübener M (2009) Experience leaves a lasting structural trace in cortical circuits. Nature 457:313-317. CrossRef Medline 
Imfeld A, Oechslin MS, Meyer M, Loenneker T, Jancke L (2009) White matter plasticity in the corticospinal tract of musicians: a diffusion tensor imaging study. Neuroimage 46:600-607. CrossRef Medline

Ishibashi T, Dakin KA, Stevens B, Lee PR, Kozlov SV, Stewart CL, Fields RD (2006) Astrocytes promote myelination in response to electrical impulses. Neuron 49:823-832. CrossRef Medline

Iwasa K, Tasaki I (1980) Mechanical changes in squid giant axons associated with production of action potentials. Biochem Biophys Res Commun 95:1328-1331. CrossRef Medline

Iwasa K, Tasaki I, Gibbons RC (1980) Swelling of nerve fibers associated with action potentials. Science 210:338-339. CrossRef Medline

Kleim JA, Markham JA, Vij K, Freese JL, Ballard DH, Greenough WT (2007) Motor learning induces astrocytic hypertrophy in the cerebellar cortex. Behav Brain Res 178:244-249. CrossRef Medline

Knott GW, Quairiaux C, Genoud C, Welker E (2002) Formation of dendritic spines with GABAergic synapses induced by whisker stimulation in adult mice. Neuron 34:265-273. CrossRef Medline

Le Bihan D (2007) The "wet mind": water and functional neuroimaging. Phys Med Biol 52:R57-R90. CrossRef Medline

Lerch JP, Yiu AP, Martinez-Canabal A, Pekar T, Bohbot VD, Frankland PW, Henkelman RM, Josselyn SA, Sled JG (2011) Maze training in mice induces MRI-detectable brain shape changes specific to the type of learning. Neuroimage 54:2086-2095. CrossRef Medline

Maguire EA, Gadian DG, Johnsrude IS, Good CD, Ashburner J, Frackowiak RS, Frith CD (2000) Navigation-related structural change in the hippocampi of taxi drivers. Proc Natl Acad Sci U S A 97:4398-4403. CrossRef Medline

Markham JA, Herting MM, Luszpak AE, Juraska JM, Greenough WT (2009) Myelination of the corpus callosum in male and female rats following complex environment housing during adulthood. Brain Res 1288:9-17. CrossRef Medline

Ming GL, Song H (2005) Adult neurogenesis in the mammalian central nervous system. Annu Rev Neurosci 28:223-250. CrossRef Medline

Pascual-Leone A, Amedi A, Fregni F, Merabet LB (2005) The plastic human brain cortex. Annu Rev Neurosci 28:377-401. CrossRef Medline

Pasternak O, Assaf Y, Intrator N, Sochen N (2008) Variational multipletensor fitting of fiber-ambiguous diffusion-weighted magnetic resonance imaging voxels. Magn Reson Imaging 26:1133-1144. CrossRef Medline

Pasternak O, Sochen N, Gur Y, Intrator N, Assaf Y (2009) Free water elimination and mapping from diffusion MRI. Magn Reson Med 62:717-730. CrossRef Medline

Paxinos G, Watson C (2005) The rat brain in stereotaxic coordinates, Ed 5. Amsterdam: Elsevier Academic.

Ransom BR, Yamate CL, Connors BW (1985) Activity-dependent shrinkage of extracellular space in rat optic nerve: a developmental study. J Neurosci 5:532-535. Medline

Rudebeck SR, Scholz J, Millington R, Rohenkohl G, Johansen-Berg H, Lee AC (2009) Fornix microstructure correlates with recollection but not familiarity memory. J Neurosci 29:14987-14992. CrossRef Medline
Sagi Y, Tavor I, Hofstetter S, Tzur-Moryosef S, Blumenfeld-Katzir T, Assaf Y (2012) Learning in the fast lane: new insights into neuroplasticity. Neuron 73:1195-1203. CrossRef Medline

Scholz J, Klein MC, Behrens TE, Johansen-Berg H (2009) Training induces changes in white-matter architecture. Nat Neurosci 12:1370-1371. CrossRef Medline

Smith SM, Nichols TE (2009) Threshold-free cluster enhancement: addressing problems of smoothing, threshold dependence and localisation in cluster inference. Neuroimage 44:83-98. CrossRef Medline

Smith SM, Jenkinson M, Woolrich MW, Beckmann CF, Behrens TE, Johansen-Berg H, Bannister PR, De Luca M, Drobnjak I, Flitney DE, Niazy RK, Saunders J, Vickers J, Zhang Y, De Stefano N, Brady JM, Matthews PM (2004) Advances in functional and structural MR image analysis and implementation as FSL. Neuroimage 23:S208-S219. CrossRef Medline

Smith SM, Jenkinson M, Johansen-Berg H, Rueckert D, Nichols TE, Mackay CE, Watkins KE, Ciccarelli O, Cader MZ, Matthews PM, Behrens TE (2006) Tract-based spatial statistics: voxelwise analysis of multi-subject diffusion data. Neuroimage 31:1487-1505. CrossRef Medline

Stevens B, Porta S, Haak LL, Gallo V, Fields RD (2002) Adenosine: a neuron-glial transmitter promoting myelination in the CNS in response to action potentials. Neuron 36:855-868. CrossRef Medline

Sutherland RJ, Rodriguez AJ (1989) The role of the fornix/fimbria and some related subcortical structures in place learning and memory. Behav Brain Res 32:265-277. CrossRef Medline

Sykova E (1997) The extracellular space in the CNS: its regulation, volume and geometry in normal and pathological neuronal function. Neuroscientist 3:28-41.

Takeuchi H, Sekiguchi A, Taki Y, Yokoyama S, Yomogida Y, Komuro N, Yamanouchi T, Suzuki S, Kawashima R (2010) Training of working memory impacts structural connectivity. J Neurosci 30:3297-3303. CrossRef Medline

Tang YY, Lu Q, Geng X, Stein EA, Yang Y, Posner MI (2010) Short-term meditation induces white matter changes in the anterior cingulate. Proc Natl Acad Sci U S A 107:15649-15652. CrossRef Medline

Tasaki I, Byrne PM (1990) Volume expansion of nonmyelinated nerve fibers during impulse conduction. Biophys J 57:633-635. CrossRef Medline

Theodosis DT, Poulain DA, Oliet SH (2008) Activity-dependent structural and functional plasticity of astrocyte-neuron interactions. Physiol Rev 88:983-1008. CrossRef Medline

Wake H, Lee PR, Fields RD (2011) Control of local protein synthesis and initial events in myelination by action potentials. Science 333:1647-1651. CrossRef Medline

Xu T, Yu X, Perlik AJ, Tobin WF, Zweig JA, Tennant K, Jones T, Zuo Y (2009) Rapid formation and selective stabilization of synapses for enduring motor memories. Nature 462:915-919. CrossRef Medline 